\title{
Trial Site Terminated by Sponsor
}

National Cancer Institute

\section{Source}

National Cancer Institute. Trial Site Terminated by Sponsor. NCI Thesaurus. Code C106576.

An indication that a clinical study was stopped at a particular site by its sponsor. 